\title{
Plasma crystal dynamics measured with a 3D plenoptic camera
}

M. Jambor, ${ }^{1}$ V. Nosenko, ${ }^{1, \text { a) }}$ S. K. Zhdanov, ${ }^{2}$ and H. M. Thomas ${ }^{1}$

1) Deutsches Zentrum für Luft- und Raumfahrt, Forschungsgruppe Komplexe Plasmen, D-82234 Weßling, Germany

2) Max-Planck-Institut für extraterrestrische Physik, D-85748 Garching, Germany

(Dated: 14 February 2016)

Three-dimensional (3D) imaging of a single-layer plasma crystal was performed using a commercial plenoptic camera. To enhance the out-of-plane oscillations of particles in the crystal, the mode-coupling instability (MCI) was triggered in it by lowering the discharge power below a threshold. 3D coordinates of all particles in the crystal were extracted from the recorded videos. All three fundamental wave modes of the plasma crystal were calculated from these data. In the out-of-plane spectrum, only the MCI-induced hot spots (corresponding to the unstable hybrid mode) were resolved. The results are in agreement with theory and show that plenoptic cameras can be used to measure the 3D dynamics of plasma crystals.

PACS numbers: 52.27.Lw, 52.35.Fp, 07.68.+m, 42.30.Va

\section{INTRODUCTION}

Complex, or dusty plasmas are suspensions of fine solid particles in a weakly ionized gas. The particles charge up by collecting electrons and ions from plasma and can be confined in the plasma regions where their weight is balanced by other forces, e.g. electrostatic force. Due to the interplay between their mutual interactions and external confinement, the particles can form various structures which are often strongly coupled and possess liquid-like or even solid-like order. The crystalline phase of complex plasma is called the plasma crystal ${ }^{1-3}$. In laboratory experiments with monodisperse particles, single-layer (2D) plasma crystals can be obtained ${ }^{4-7}$.

Complex plasmas are popular versatile model systems that are used to simulate the structure and dynamic processes in regular liquids and solids. With microparticles serving as proxy "atoms," they offer the benefit of realtime direct imaging of various processes at the "atomistic" level. Examples include the studies of waves ${ }^{5-8}$, phase transitions $^{9-11}$, and transport phenomena ${ }^{12-16}$ in complex plasmas.

Direct imaging of particles is a primary diagnostic in experiments with complex plasmas. Most often, a cross section of the particle suspension is illuminated with a laser sheet and imaged with a video camera. This method has obvious limitations. A full 3D imaging technique is highly desirable even in the experiments with single-layer plasma crystals.

Various 3D imaging methods have been used in complex plasma experiments. Stereoscopic imaging is a well-established method to obtain 3D coordinates of particles ${ }^{17,18}$. In the digital in-line holography technique, the depth information is acquired from diffraction patterns produced by individual particles ${ }^{19,20}$. In the colorgradient method, the depth information is encoded in the color of particle images ${ }^{21}$. Tomographic methods use

\footnotetext{
a)Electronic mail: V.Nosenko@dlr.de.
}

moving mirrors ${ }^{22}$ or cameras ${ }^{23}$ to reconstruct the structure of a 3D complex plasma. The latter method measures the dust particle density without resolving individual particle positions.

Recently, a method of evaluating 3D velocities of particles in a single-layer plasma crystal was proposed where the depth information is acquired from the particle image intensity variations ${ }^{24}$. This method is limited to smallamplitude out-of-plane displacements and requires elaborate calibration due to the nonlinear intensity profile of the illuminating laser sheet.

In the present study, we explore a new approach to 3D imaging of particles by using a plenoptic camera that allows a direct detection of particle motion in all three spatial directions in a single measurement with a single camera.

Plenoptic cameras, which were proposed theoretically more than 100 years $\mathrm{ago}^{25}$, are lately emerging on the market, as digital photography, image processing, and methods of microfabrication have registered remarkable improvements in the last decade. These cameras immediately caught the attention of the complex plasma community. Hartmann et al. ${ }^{26}$ first demonstrated the possibility to use a plenoptic camera for 3D particle detection in complex plasmas, by producing still images of plasma clusters with about 60 particles.

We used a commercial Raytrix R5 plenoptic camera ${ }^{27}$ to image an extended monolayer plasma crystal in order to study its wave modes in the regime of mode coupling instability $(\mathrm{MCI})^{28}$. In this paper, we present the results of these investigations.

In Sec. II, we will discuss the theory of plenoptic cameras in a detailed fashion. Sec. III will present our experimental setup. Our analysis method will be outlined in Sec. IV. Finally, the results will be discussed and summarized in Sec. V. 


\section{PLENOPTIC CAMERAS}

The difference in the hardware of a plenoptic camera compared to a standard camera is only one element, which is a microlens array that is placed in front of the camera chip. However, this relatively small difference changes the capabilities of the camera significantly and turns it into a 3D camera, as we will discuss in the following.

While in a conventional camera the depth information is lost, if not encoded indirectly in the scene (compare the method presented $\mathrm{in}^{24}$ ), the microlenses in a plenoptic camera subsample the main lens image onto the camera sensor in a way that each object point appears in several micro-images on the sensor. Thereby, the distance from the sensor and the focal lengths of the microlenses are crucial parameters, since they allow two different working modes, the Keplerian and Galilean modes ${ }^{29}$.

In the first case, the microlenses were placed exactly their focal length away from the camera sensor. Thus, they are themselves focused to infinity and each pixel behind a microlens captures a different subset of the main lens aperture, while pixels in other microlens images will capture the same subset from a slightly different angle. Early setups of plenoptic cameras practiced this working principle $^{30,31}$.

Recent designs, such as the Raytrix R5 camera that we have been using, employ the Galilean mode, where the microlenses are focused onto the main lens image and their distance to the sensor does not equal their focal length ${ }^{32,33}$. In the following, we focus on this principle. The geometrical arrangement of a Galilean plenoptic camera is shown in the upper panel of Figure 1, for the case that the main lens image lies behind the microlens array (virtual image).

Let $f_{m}$ be the focal length of one microlens, $D_{m}$ its diameter, and $B_{m}$ its distance to the sensor. The main lens diameter shall be $D_{L}$, and its distance to the sensor $B_{L}$. The distance between the microlens and the main lens image is denoted as $a$. The sign of this distance is defined in a way that $a$ is positive when a real main lens image is regarded, and negative when a virtual main lens image is regarded. The ratio of $a$ and $B_{m}$ is referred to as virtual depth ${ }^{33}$, and is the inverse magnification of the microlens:

$$
v=\frac{a}{B_{m}}
$$

As can be seen in Figure 1, the microlenses subsample the main lens image onto the sensor. We can utilize the so-called light field ${ }^{34}$ in order to describe the subsampling quantitatively. The light field gives the radiance in free space behind an optical system as a function of lateral position and direction of ray incidence. Thus, it is a four-dimensional vector, but can be projected to two dimensions for further theoretical discussions without the loss of generality ${ }^{31,32}$. For further explanations, let us

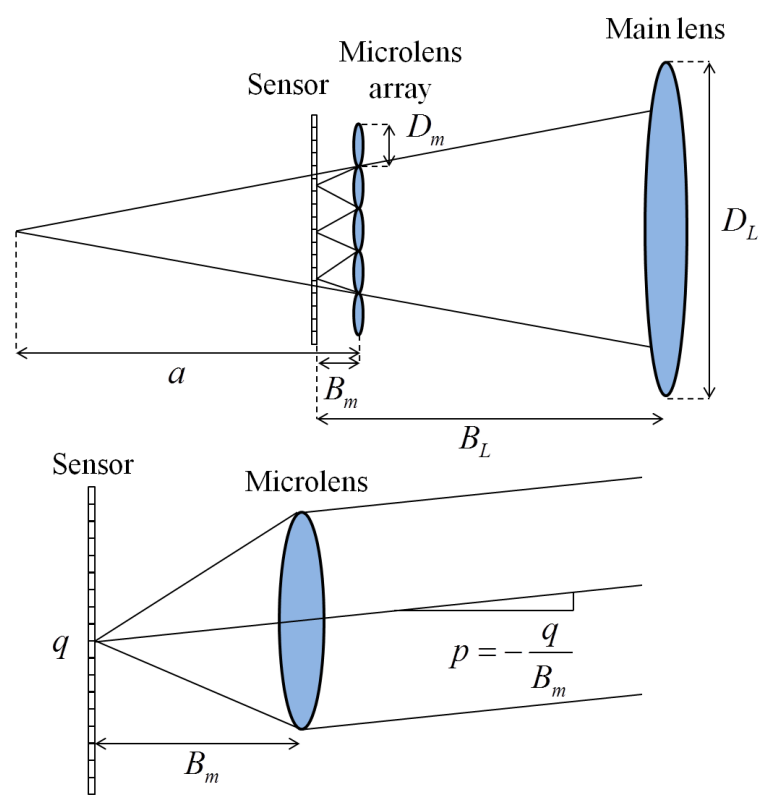

FIG. 1. (color online) Upper panel: Geometrical arrangement of a Galilean plenoptic camera. The sensor is located at a distance $B_{m}$ behind the microlens array and a distance $B_{L}$ behind the main lens. The diameters of the microlenses and the main lens are $D_{m}$ and $D_{L}$, respectively. The main lens image forms at a distance $a$ behind the microlenses. Lower panel: Geometry of a single microlens system in a Galilean plenoptic camera. The microlens is placed at a distance $B_{m}$ in front of the sensor. For a point with sensor coordinate $q$, the direction of incidence of the central ray is $p=-q / B_{m}$. The two parameters uniquely define any ray impinging on the sensor. See also ${ }^{32}$.

employ a $2 \mathrm{D}$ light field $L(q, p)$, where $q$ is the lateral coordinate on the sensor and $p$ the direction of incidence of rays impinging onto that coordinate ${ }^{32}$, see the lower panel in Figure 1.

Irradiation $I$ at any given point in $q$-space can be calculated as the integral over all directions of impingence:

$$
I(q)=\int L(q, p) d p
$$

Thus, the intensity $I(q)$ for all possible coordinates $q$ gives the complete image.

For a plenoptic camera operating in Galilean mode, the irradiation onto a pixel with coordinate $q$ on the sensor plane with respect to the optical axis of the microlens at $q=0$ is $^{32}$ :

$$
I_{s}(q)=\int L_{s}(q, p) d p=\frac{D_{m}}{B_{m}} L_{a}\left(-v q, \frac{1}{B_{m}} q\right),
$$

where $L_{a}(q, p)$ is the light field at the main lens image plane and $L_{s}(q, p)$ the light field at the sensor plane ${ }^{32}$. According to Eq. (3), each microlens images a subsection of the main lens image with scaling $1 /|v|$ onto the 


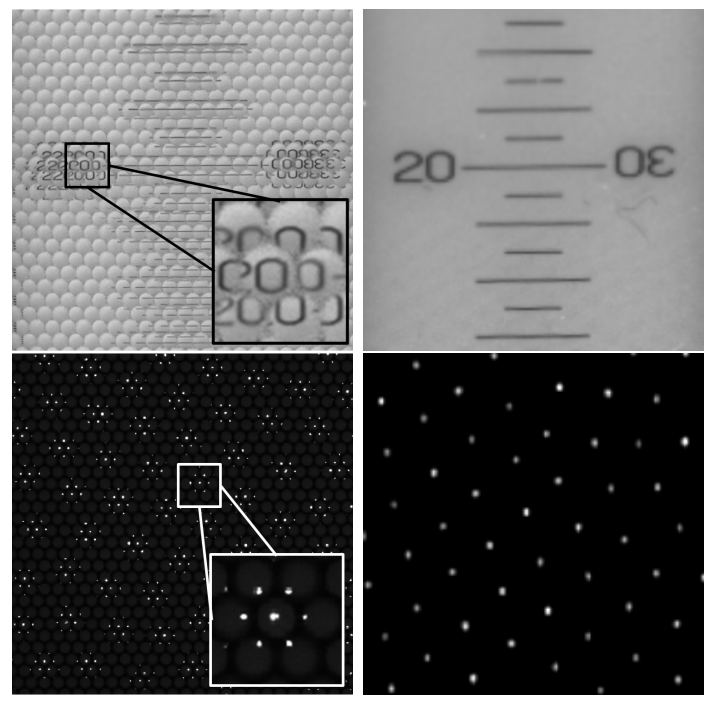

FIG. 2. Examples of images captured by the plenoptic camera. Top panels: raw image (left) and reconstructed focused image (right) of a test chart, bottom panel: raw image (left) and reconstructed focused image (right) of particles in a $2 \mathrm{D}$ plasma crystal. As can be seen in the raw images, each object point is visible in several microimages subsampled by the microlens array (see enlarged sections in boxes), allowing for triangulation and depth estimation.

sensor area behind itself. Hence, the lateral resolution of a Galilean plenoptic camera is the chip resolution scaled by this factor.

The subsampling of the main lens image by microlenses makes the raw image rather involved. Exemplary raw images of objects captured with the Raytrix R5 camera are shown in Figure 2 (left column). In the upper panel, a test target is imaged, whereas in the lower panel, one can see particles in a 2D plasma crystal.

As each object point is sampled onto several microimages on the chip, it is possible to perform triangulation in order to calculate the depth of the corresponding point. For such triangulation, an identification of similar object points imaged in different microlenses has to be carried out. The usual approach for that is to calculate the sum of absolute intensity differences along pixel patches within the microimages ${ }^{33,35}$, which requires sufficient contrast, as can be found at the edges of structures.

When a consistent point is found in at least two microlenses, triangulation provides an estimate of the depth of that point. It can be derived regarding geometrical optics of the microlens subsystem.

Let us adopt a general model from ${ }^{33}$, consisting of two microlenses with the main lens image behind the sensor and the optical axis of the main lens in the middle between them. The model is sketched in Figure 3. The distance from the optical axis to the microlens's center is expressed as $k D_{m} / 2$, a function of the lens diameter $D_{m}$ and a factor $k$, by which the lens radius has to be multiplied in order to obtain the distance from a lens's center to its neighbours of different order.

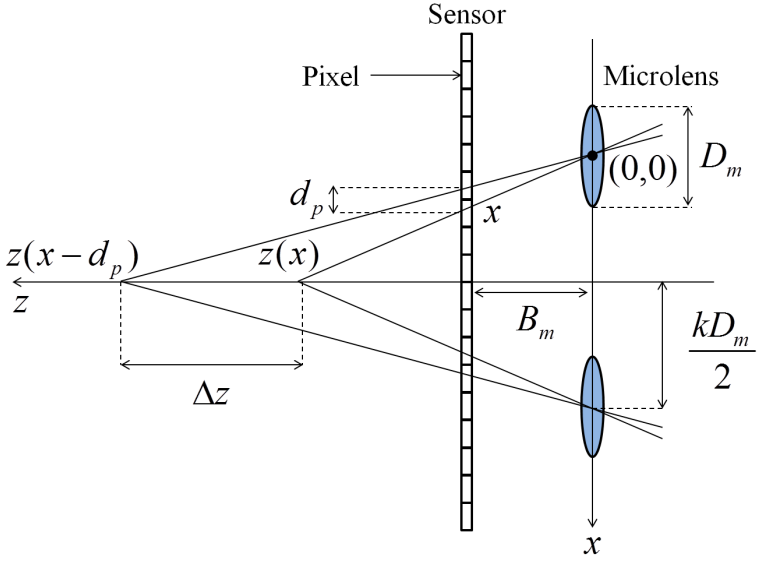

FIG. 3. (color online) Geometrical relations for triangulation. The image-side depth coordinate $z(x)$ with respect to the origin at the microlens' center can be calculated as the point where the central light ray through this microlens intersects with a ray that emerged from the same object point, but passed a different microlens. A light ray that passes the sensor with a shift of the pixel size $d_{p}$ in $x$-direction will intersect with its counterpart at depth $z\left(x-d_{p}\right)$. Hence, the lateral resolution, given by the pixel size $d_{p}$ in this figure, results in a finite depth resolution $\Delta z$ along the optical axis. See also ${ }^{33}$.

As a point needs to appear in at least two microlenses, this limits the factor $k$ to a minimum value of $k_{\min }=2$ in our simplified 1D model. This in return means, that the distance between the main lens image plane and the microlens array may not be closer than $k_{\min } B_{m}$.

In the case of a hexagonal 2D microlens array, as featured in our plenoptic camera, the virtual depth (see equation 1) is limited to a minimum value of $|v|_{\min }=$ $2 \sqrt{1+\tan ^{2}(\pi / 6)}=4 / \sqrt{3} \approx 2.31$.

Given the distance between the sensor and the microlenses $B_{m}$, the pixel size $d_{p}$, and the position of one distinct point in a microimage $x$, a relationship for the depth coordinate $z$ can be deduced:

$$
\frac{B_{m}}{x}=\frac{2 z}{k D_{m}} \Rightarrow z(x)=\frac{k B_{m} D_{m}}{2 x} .
$$

For a neighbouring pixel in the same microimage, the lateral position will be $x-d_{p}$ and the corresponding depth will be $z\left(x-d_{p}\right)$, resulting in an image depth uncertainty of

$$
\Delta z=\left|z(x)-z\left(x-d_{p}\right)\right| .
$$

The model takes the pixel size into account as the lateral resolution. This is a reasonable assumption when it comes to the imaging of extended objects. Modern particle detection algorithms ${ }^{36}$, however, are able to overcome that limitation and detect lateral particle positions with higher accuracy, up to subpixel resolution. 
As lateral and depth resolution of the image are correlated, a more accurate depth estimation for particles is possible in this case. Moreover, triangulation can be performed for every pair of microimages in which a particle appears. Comparing the different lens combinations, the image depth can be calculated even more precisely using epipolar geometry ${ }^{37}$.

Ultimately, the main lens used for imaging sets the restraints for lateral and depth resolution. It is well known, that an object that is imaged by an optical system is sharpest when it is in focus. Shifting the object away from that position will give its image a defocused blur. The range, in which the blurring is still acceptable, is referred to as the depth-of-field (DOF), where the term "acceptable" is mostly defined by a blur smaller than the pixel size ${ }^{29}$.

It is worth mentioning that plenoptic cameras generally have extended DOF's compared to conventional cameras. The Raytrix R5 employs a hexagonal microlens array with lenses of three different focal lengths in order to extend the DOF, while maintaining highest possible lateral resolution ${ }^{33}$. As a special feature of plenoptic cameras, the image can be refocused within the DOF after it has been recorded using light field rendering and image synthesization. These special characteristics are of little use in experiments with 2D plasma crystals, but may be beneficial for 3D complex plasmas, where different layers of a crystal could be brought into focus and analyzed separately, all for a single recording.

\section{EXPERIMENTAL METHOD}

Our experimental setup is shown schematically in Figure 4. We used a modified Gaseous Electronics Conference (GEC) radio-frequency (rf) reference cell ${ }^{4}$. A Dressler Cesar rf generator was connected via a blocking capacitor to the electrode in the chamber and sustained an argon rf discharge. Spherical melamine-formaldehyde (MF) particles with a diameter of $9.19 \pm 0.09 \mu \mathrm{m}$ and a mass of $m=6.15 \times 10^{-13} \mathrm{~kg}$ were injected from a particle dispenser and suspended in the sheath region of the plasma, located above the lower rf electrode.

A highly purified single layer of particles was prepared using the following method. The particles were suspended in the plasma at $10 \mathrm{~W}$ rf discharge power. After suspension, the particles crystallized at the argon pressure of 1.5 $\mathrm{Pa}$. The suspension was then purified in a common way by a stepwise decrease of the discharge power until the confinement was weak enough that particles levitating beneath the main layer (e.g., agglomerations) dropped to the electrode ${ }^{38,39}$. Using the side-view camera, we verified that there were no particles levitating above the main layer ${ }^{40}$.

After the pure single layer was established, the rf power was restored to its original value of $10 \mathrm{~W}$ and the pressure was set to $0.8 \mathrm{~Pa}$. As the next step, the transition toward the mode-coupling regime was undertaken by an-
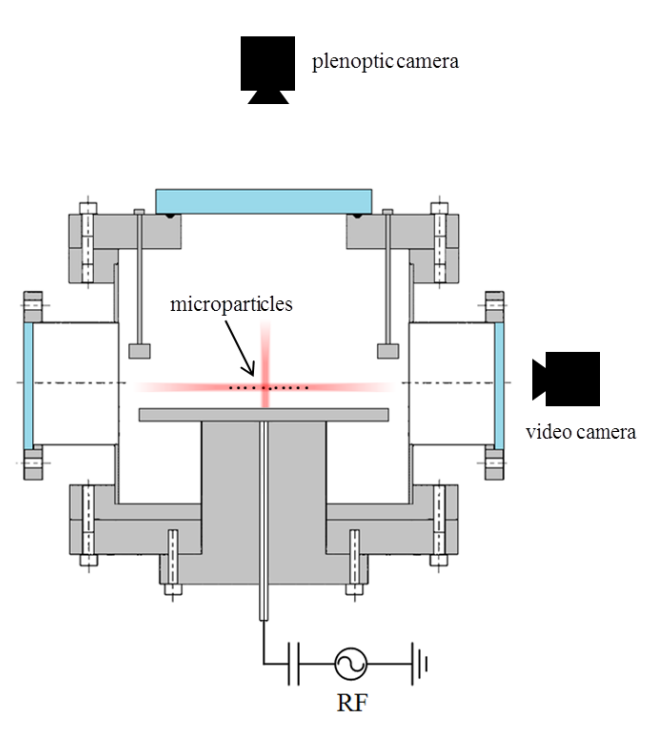

FIG. 4. (color online) Experimental setup. We used a modified GEC rf reference cell and suspended MF microspheres with a diameter of $9.19 \pm 0.09 \mu \mathrm{m}$ above the lower rf electrode in the (pre)sheath region of an argon plasma. The particle levitation height is approximately $1 \mathrm{~cm}$ above the $\mathrm{rf}$ electrode. The particles were illuminated by a horizontal 660 -nm laser sheet and imaged from the top with the Raytrix R5 plenoptic camera. Additionally, a stripe of particles was illuminated with a vertical 635 -nm laser sheet and imaged from the side with the Photron FASTCAM 1024 PCI video camera.

other stepwise decrease of the rf power in order to weaken the confinement. As mode coupling will cause the crystal to melt at sufficiently low pressures and discharge powers, the melting point had to be found iteratively.

After melting had been observed for the first time, the experimental conditions were restored to the values just above the critical point. For the main experiment, a discharge power of $1 \mathrm{~W}$ at a pressure $p=0.8 \mathrm{~Pa}$ was used.

The particles were illuminated by horizontal and vertical laser sheets with the wavelengths of $660 \mathrm{~nm}$ and $635 \mathrm{~nm}$, respectively. Corresponding bandpass interference filters were used in the two video cameras imaging the suspension from the top and from the side, respectively, the plenoptic Raytrix R5 and conventional Photron FASTCAM 1024 PCI. The powers of the illuminating lasers were $100 \mathrm{~mW}$ each.

The Raytrix R5 camera was mounted above the chamber at a working distance of $0.44 \mathrm{~m}$ from the particle suspension, and was equipped with a Zeiss Makro-Planar $\mathrm{T}^{* 2 / 100}$ lens. The lens aperture was matched to the microlenses' aperture of $f / 2.4$, see $^{31,33}$. The frame rate was 60 frames per second (fps), with an exposure time of $0.015 \mathrm{~s}$. The frame rate was limited by poor illumination above $60 \mathrm{fps}$, that compromised the particle tracking. 


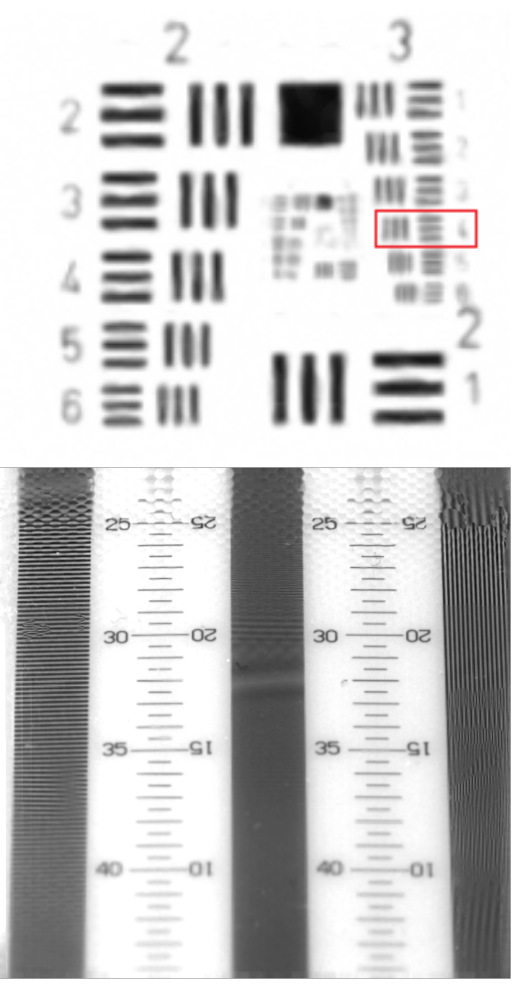

FIG. 5. (color online) Test targets imaged with the Raytrix R5 camera. Top panel: 1951 USAF test target. The smallest resolved structure is the 4th element of the 3rd group (indicated by a rectangle), corresponding to a line width of $44.25 \mu \mathrm{m}$. Bottom panel: DOF $5-15$ test target imaged in the $45^{\circ}$ orientation compared to the optical axis. Line patterns with $5 \mathrm{lp} / \mathrm{mm}$ and $15 \mathrm{lp} / \mathrm{mm}$ along the $z$-axis are featured. $5 \mathrm{lp} / \mathrm{mm}$ can be resolved, corresponding to a line width of $100 \mu \mathrm{m}$. The depth-of-field (object side) is $15 \pm 0.4 \mathrm{~mm}$ (uncertainty of \pm 2 lines).

We determined the camera's optical lateral and depth resolution, as well as the object-side DOF using the common test targets 1951 USAF and DOF 5-15. The first one is imaged orthogonally, while the latter draws an angle of $45^{\circ}$ with the optical axis. Figure 5 shows the results of these tests.

The lateral resolution can be approximated as $44 \mu \mathrm{m}$, as the 4th element of the 3rd group of the 1951 USAF test chart is still resolved in the top panel of Figure 5. The depth resolution was estimated using the DOF 5-15 target, which features line patterns at frequencies of 5 line pairs per millimeter $(\mathrm{lp} / \mathrm{mm})$ and $15 \mathrm{lp} / \mathrm{mm}$. As the $5 \mathrm{lp} / \mathrm{mm}$ pattern could be resolved in the lower panel of Figure 5, we take into account its line width of $100 \mu \mathrm{m}$ as a depth resolution benchmark. As this remains constant over a range of approximately $15 \mathrm{~mm}$, this value will serve as a benchmark for the depth-of-field. An uncertainty of \pm 2 lines $( \pm 0.4 \mathrm{~mm})$ is taken into account.

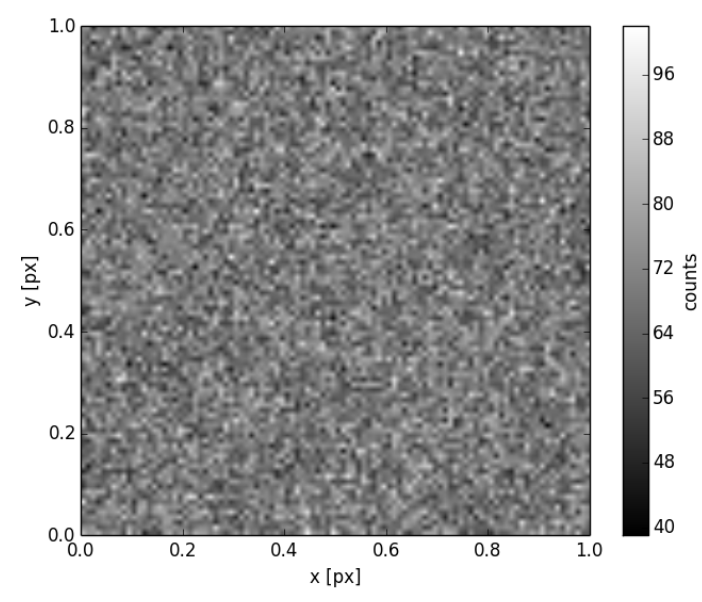

FIG. 6. Map of the fractional parts of calculated particle positions from a 400-frames video recorded during the MCImelting experiment. The more or less uniform distribution of the data points means that pixel locking is mild, if any.

\section{ACCURACY OF PARTICLE POSITION MEASUREMENT}

An experimental video with particles was analyzed to identify and trace them from frame to frame. We used the proprietary tracking software RxFlow (provided by the camera manufacturer), which is based on the estimates of the center-of-intensity for every particle image. The accuracy of particle position measurement is related to the camera's optical resolution discussed above, but is not equal to it. According to the manufacturer ${ }^{41}$, the lateral and depth resolution of particle detection are respectively $\pm 0.1 \%$ and $\pm 1 \%$ of the camera's DOF. As the DOF is $15 \mathrm{~mm}$ in our case, this yields $15 \mu \mathrm{m}$ for the lateral and $150 \mu \mathrm{m}$ for the depth resolution of particle detection.

The particle identification method used provides subpixel resolution. This brings about a question on the possible presence of pixel locking in calculated particle positions. Pixel locking is an artifact by which calculated particle positions are not uniformly distributed inside every pixel, but rather concentrate at certain values relative to the pixel edges ${ }^{42}$, or a tendency of particle position estimates to cluster around integer values ${ }^{43-45}$. A practical way to evaluate pixel locking in a given measurement is to plot the particle positions in a map of their fractional parts $\operatorname{frac}(y) \mapsto \operatorname{frac}(x)$. A strong clustering of the data points in such a map will indicate pixel locking.

The fractional parts of all particle positions obtained from a 400-frames video recorded during the MCImelting experiment are shown in Fig. 6. The clustering of data points in this figure is weak to moderate. An error of $\simeq 23 \%$ stemming from pixel locking is estimated in a standard way calculating the variance of the fractional part distribution. 
We noticed that sometimes a spurious particle would appear in the tracking data above or beneath a real particle. The side-view videos clearly confirmed that these particles were artifacts. There are two methods to solve this problem. The first method is to set a threshold for the number of microimages in which a particle has to appear in order to be taken into account. Setting the threshold to a minimum value of 4 microimages eliminated the spurious particles. The second method is to entirely disregard one of the three types of microlenses during the particle tracking since one microlens type is significantly different from the other two, according to the manufacturer ${ }^{41}$. Both methods were found empirically and work equally well. As the second method was recommended by the manufacturer, it was used to filter the data for further processing.

Figure 7 shows the trajectories of representative particles from the MCI-melting experiment. The upper panel shows the 3D trajectory of a single particle. Time is indicated by color coding. In the lower panel, we show the $z$ coordinate of this particle as a function of time and compare it to another representative particle from the same experiment, imaged with the (regular) side-view camera. In the latter case, the particle positions were detected from the side view video sequence using a simple particle tracking algorithm based on the particle image centerof-intensity calculation, see e.g. ${ }^{46}$. Both measurements reveal a vertical oscillation amplitude of approximately $0.15 \mathrm{~mm}$.

\section{SPECTRAL ANALYSIS OF PARTICLE VELOCITY FLUCTUATIONS}

The particle tracking data was subjected to further analysis. As our observations show, the particles exhibit seemingly random motion around their equilibrium positions both in the horizontal (in-plane) and vertical (out-of-plane) directions. The amplitude of vertical oscillations is larger than that of horizontal oscillations, especially in an active (marginally unstable) crystal; see Figure 7, where both cameras measured the vertical oscillation amplitude of $\approx 0.15 \mathrm{~mm}$. The particle oscillations result in the appearance of the so-called naturally occurring waves, a prominent and easily detectable feature of the crystal dynamics. Therefore, it is instructive to use them to explore crystal properties such as, e.g., phonon speed $^{5,7,47-49}$.

To study the spectral characteristics of the particle random motion, we Fourier transform the particle velocity field components $\mathbf{V}(x, y, z)=\{V x, V y, V z\}$ both in space $\{x, y\}$ and time $t$, introducing a Fouriertransformed velocity field $\mathbf{V}_{\omega, \mathbf{k}}$, where $\omega$ is the circular frequency and $\mathbf{k}=\left\{k_{x}, k_{y}\right\}$ is the in-plane wave vector. This is a rather standard procedure allowing one to obtain the velocity fluctuation spectra $I_{\omega, \mathbf{k}} \propto\left|\mathbf{V}_{\omega, \mathbf{k}}\right|^{2}$; see, e.g. ${ }^{50}$. In our geometry of a single layer (or an elastic membrane where $3 \mathrm{D}$ displacements are allowed), to
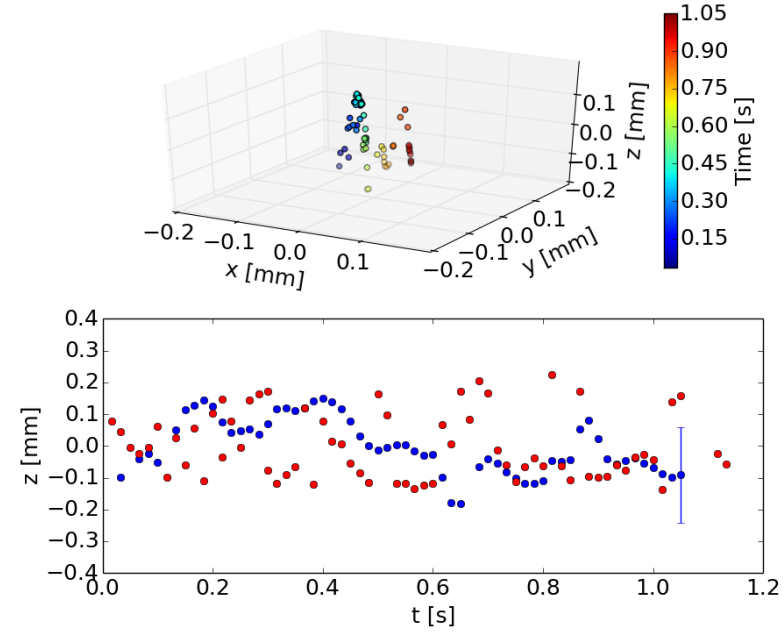

FIG. 7. (color) 3D trajectory of a single particle from the region where MCI set in, recorded by the plenoptic camera (upper panel). The time interval is $1.05 \mathrm{~s}$ and is color-coded from blue to red. The particle's $z$ (depth) coordinate as a function of time is shown in the lower panel by blue dots. The accuracy of the measurement $( \pm 150 \mu \mathrm{m})$ is indicated by error bars. For comparison, we show the trajectory of another representative particle from the same region imaged with a regular side-view camera (red dots). The frame rate was $60 \mathrm{fps}$ for both plenoptic and regular cameras.

characterize the distribution of the wave energy over its components it is convenient to introduce two in-plane orthogonal unit vectors $\mathbf{e}_{\mathbf{k}}=\mathbf{k} /|\mathbf{k}|, \mathbf{e}_{\mathbf{k}}^{\tau}=\mathbf{e}_{\mathbf{k}} \times \mathbf{e}_{z}$, and the out-of-plane unit vector $\mathbf{e}_{z}$ perpendicular to the layer's plane. Note that both, in-plane and out-of-plane particle oscillations, are activated. Therefore, the spectral intensities of in-plane longitudinal $I_{\omega, \mathbf{k}}^{(L)}$, transverse $I_{\omega, \mathbf{k}}^{(T)}$, and out-of-plane $I_{\omega, \mathbf{k}}^{(Z)}$ particle movements, which contribute to the total intensity $I_{\omega, \mathbf{k}}$, are of great interest:

$$
I_{\omega, \mathbf{k}}=I_{\omega, \mathbf{k}}^{(L)}+I_{\omega, \mathbf{k}}^{(T)}+I_{\omega, \mathbf{k}}^{(Z)}, \quad I_{\omega, \mathbf{k}}^{(L, T, Z)} \propto\left|\mathbf{V}_{\omega, \mathbf{k}}^{(L, T, Z)}\right|^{2},
$$

where $\mathbf{V}_{\omega, \mathbf{k}}^{(L)}=\mathbf{e}_{\mathbf{k}}\left(\mathbf{e}_{\mathbf{k}} \mathbf{V}_{\omega, \mathbf{k}}\right), \mathbf{V}_{\omega, \mathbf{k}}^{(T)}=\mathbf{e}_{\mathbf{k}}^{\tau}\left(\mathbf{e}_{\mathbf{k}}^{\tau} \mathbf{V}_{\omega, \mathbf{k}}\right), \mathbf{V}_{\omega, \mathbf{k}}^{(Z)}=$ $\mathbf{e}_{z}\left(\mathbf{e}_{z} \mathbf{V}_{\omega, \mathbf{k}}\right)$ are the longitudinal, transverse, and out-ofplane spectral components of the particle velocity field, respectively.

The distributions of all three components of the wave energy are shown in Figure 8. The fine-structured wave energy distributions indicate the existence of the wellresolved wave branches which, judging by their narrow width, are resonant in nature. The physics of these wave branches is not difficult to address ${ }^{49,51,52}$. For instance, the out-of-plane long-wavelength oscillations is an optical-like collective mode. In fact, the particles oscillate at a nearly constant angular frequency defined by the inhomogeneity length $L_{E}$ of the vertical electric field E: 

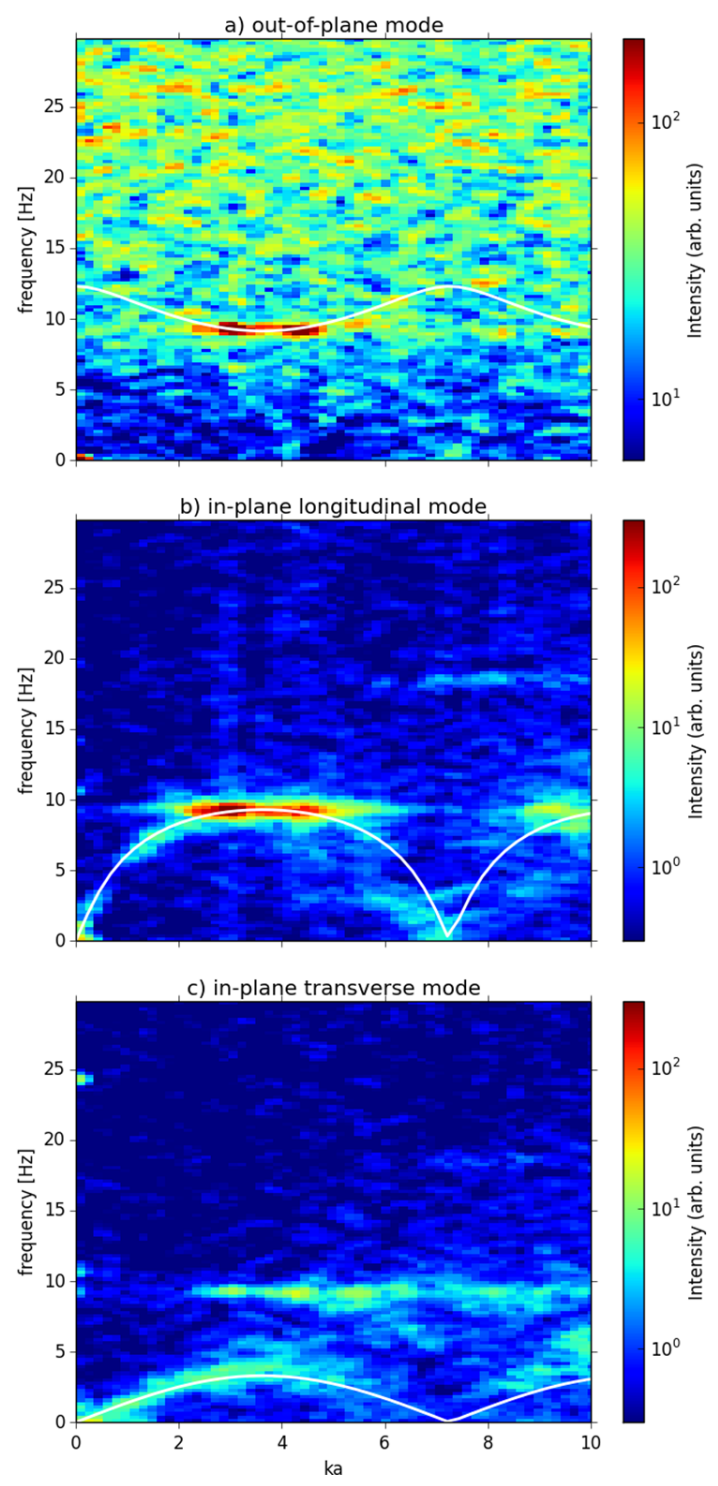

FIG. 8. (color) Fluctuation spectra of particle velocity for the (a) out-of-plane mode, (b) longitudinal and (c) transverse in-plane modes. Notice the hot spots at the hybrid frequency $f_{\text {hyb }}=9.25 \pm 1.25 \mathrm{~Hz}$ and $k a=3.0 \pm 0.5$ and $4.3 \pm 0.5 \mathrm{in}$ the out-of-plane and longitudinal spectra, indicating the occurrence of mode coupling. The wave vector $\mathbf{k}$ points along one of the main crystallographic axes ${ }^{50}$. White lines show theoretical dispersion relations ${ }^{7}$.

$$
\omega^{2} \approx \Omega^{2}=g / L_{E}, \quad L_{E}=\left|E / E^{\prime}\right|,
$$

where $g$ is the gravity acceleration. The propagation speed of these waves is very low ${ }^{51}$. The long-wavelength compressions as well as shear fluctuations obey the acoustic dispersion relations:

$$
\omega_{L, T}^{2}=C_{L, T}^{2} k^{2}
$$

where $k$ is the wave number, and $C_{L, T}$ the longitudinal and transverse sound speeds, respectively. The latter are given by the following relationships:

$$
C_{L}=\xi_{L}(\kappa) C_{Q}, \quad C_{T}=\xi_{T}(\kappa) C_{Q}, \quad C_{Q}=|Q| /(M a)^{1 / 2},
$$

where $Q$ is the particle charge and $M$ the particle mass. The coefficients $\xi_{L, T}$ are dependent on the so-called interaction range, or shielding parameter $\kappa=a / \lambda_{D}$, the ratio of the crystal constant $a$ to the plasma screening length $\lambda_{D}$. The functional dependencies $\xi_{L, T}=\xi_{L, T}(\kappa)$ are well-known (see, e.g., ${ }^{7}$ ). In particular, for $\kappa<2$, which range is represented most often in the experiments, they are extremely simple:

$$
\xi_{T}(\kappa) \approx \text { const, } \quad \xi_{L}(\kappa) \propto \kappa^{-1 / 2}
$$

and therefore the relationships in Eq. (9) are commonly used as a diagnostic tool to measure the particle charge and the interaction range of the plasma crystal ${ }^{5,47}$.

In our case, this procedure yields the following parameters: $C_{L}=(38.2 \pm 3.8) \mathrm{mm} / \mathrm{s}, C_{T}=(6.4 \pm 0.6)$ $\mathrm{mm} / \mathrm{s}, a=(0.72 \pm 0.05) \mathrm{mm}, \lambda_{D}=(1.04 \pm 0.45) \mathrm{mm}$, $\kappa=0.69_{-0.2}^{+0.3}$, and $Q=\left(17600_{-1800}^{+2000}\right) e$.

By virtue of Eq. (9), the long-wavelength in-plane fluctuations are only weakly dispersive, because their phase velocities $V_{\mathrm{ph}}$ are almost independent of $k$ :

$$
\left(V_{\mathrm{ph}}\right)_{T, L}=(\omega / k)_{T, L}=C_{T, L}
$$

whereas the out-of-plane waves, by Eq. (7), are always strongly dispersive:

$$
\left(V_{\mathrm{ph}}\right)_{Z}=(\Omega / k) \propto k^{-1}
$$

In the domain of short wavelengths, all three wave modes are strongly dispersive. Dispersion of the waves is mainly caused by the discreteness of the lattice layer. Since no simple analytical formulae exist describing the wave dispersion, one needs to sum up over many elementary crystal cells to compute the accurate dispersion relations; see for instance ${ }^{7,49,53}$. To make an example, the dispersion curves of in-plane and out-of-plane velocity fluctuations calculated with the given above values of $a, \kappa$, and $Q$ are shown in Figure 8. They agree remarkably well with our experimental spectra.

To analyze the hot spots in Figure 8 quantitatively, we averaged the spectral intensity over a frequency range of $9.25 \pm 1.25 \mathrm{~Hz}$, see lower panels in Figure 9. The spectral intensity has two distinct peaks at $k a=3.0 \pm 0.5$ and $k a=4.3 \pm 0.5$ located symmetrically around the border of the first Brillouin zone at $k a=2 \pi / \sqrt{3}$. This is also in excellent agreement with theory ${ }^{54}$. 

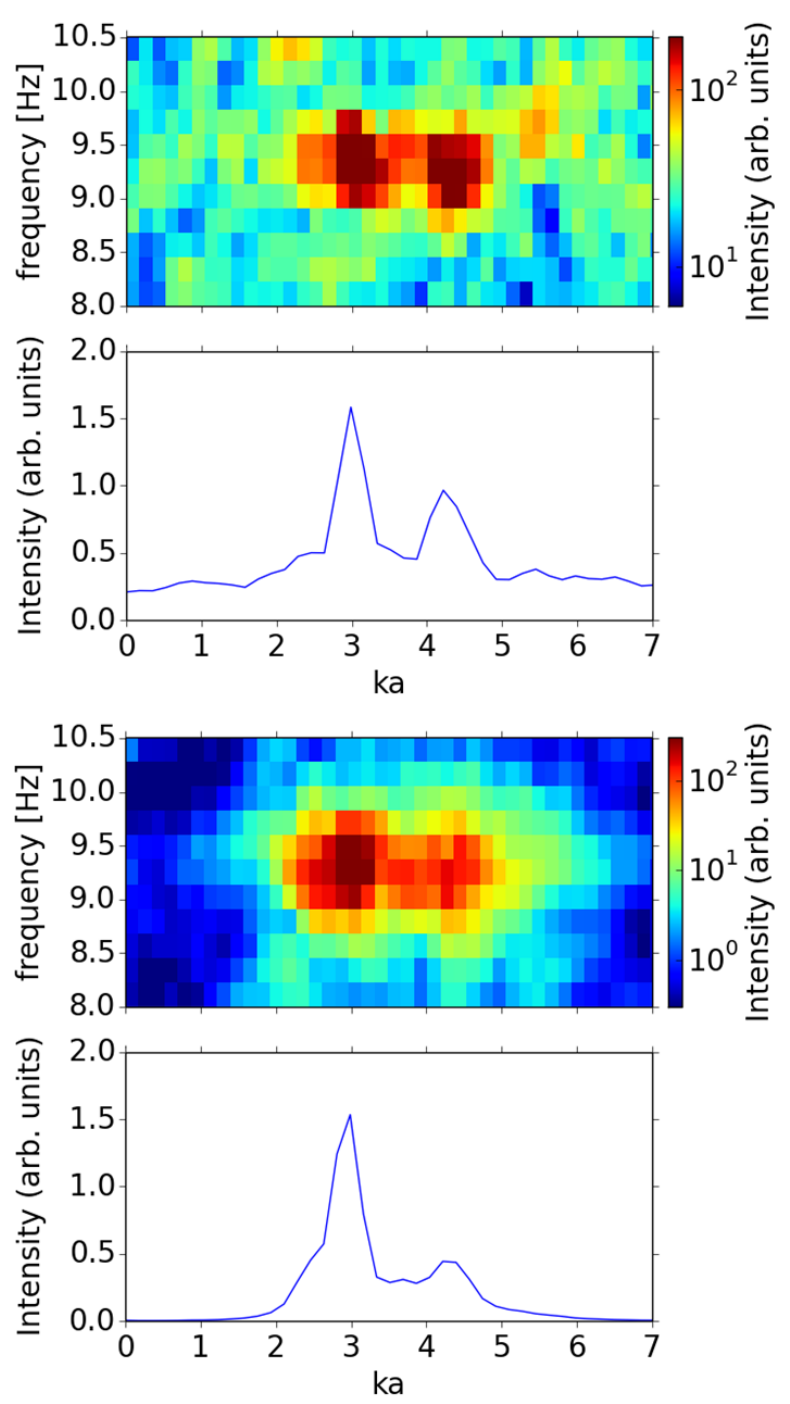

FIG. 9. (color online) Zoom-in on the hot spots from Figure 8. The upper two panels show the fluctuation spectrum of particle velocity and the spectral intensity profiles averaged over a frequency range of $9.25 \pm 1.25 \mathrm{~Hz}$ for the out-of-plane mode. The lower two panels show the same for the longitudinal mode. The spectral intensity has distinct peaks at $k a=3.0 \pm 0.5$ and $4.3 \pm 0.5$. Note that the hot spot outside of the first Brillouin zone is weaker in both cases.

\section{DISCUSSION AND CONCLUSIONS}

Full 3D trajectories of particles in a single-layer plasma crystal were measured using one plenoptic camera capturing the top view of the crystal. To enhance the vertical (out-of-plane) oscillations of particles, the measurements were carried out in the regime of mode-coupling instability. From the measured particle trajectories, the velocity fluctuation spectra of all three fundamental wave modes of the plasma crystal were calculated: longitudinal inplane, transverse in-plane, and the out-of-plane mode. Since the measurements were carried out in the mode- coupling regime, an unstable hybrid mode appeared at the intersection of the longitudinal in-plane and out-ofplane modes ${ }^{28}$. The hybrid mode manifested itself as two areas with very high spectral intensity in the vicinity of the mode intersections, referred to as hot spots, in the fluctuation spectra of particle velocity, see Figure 8 . The two hot spots were individually resolved, see Figure 9. They are located close to the border of the first Brillouin zone, in accordance with theory ${ }^{54}$ and previous observations $^{50}$. The complete dispersion relations of the longitudinal and transverse in-plane modes are also in excellent agreement with theoretical curves calculated from the crystal parameters. We note that a careful analysis of the shape of the 3D trajectory in Figure 7 can help to experimentally prove the theoretical prediction that the particle trajectories in the hybrid mode are inclined ellipses ${ }^{55}$; this study is reserved for the future work.

Fluctuation spectra of the particle $z$-velocity (along the line of sight) in the MCI regime revealed the unstable hybrid mode (hot spots) but the rest of the out-of-plane dispersion relation was not resolved. The amplitude of particle oscillations $A_{z}$ during MCI was measured to be around $150 \mu \mathrm{m}$. In a separate test where MCI was not triggered in the plasma crystal, $A_{z}$ was much smaller and the out-of-plane dispersion relation was not resolved at all. Therefore, we conclude that the particle oscillations along the camera's line of sight can be resolved if their amplitude is at least $150 \mu \mathrm{m}$. This compares well with the accuracy of the particle $z$ coordinate given by the camera's manufacturer, see Section IV. The particle $x, y$ coordinates were determined with the accuracy of $15 \mu \mathrm{m}$, which led to the fully resolved dispersion relations of the in-plane modes.

To summarize, we demonstrated the use of a plenoptic camera for 3D particle tracking velocimetry in a singlelayer plasma crystal during mode-coupling instability. An important advantage of this method is that it allows to measure the full 3D trajectories of particles from a video taken by a single plenoptic camera. Although previous studies have already shown the potential of plenoptic cameras for particle detection in complex plasmas ${ }^{26}$, here we showed for the first time that plenoptic cameras can be used to measure 3D dynamics of particles in extended complex plasma systems.

\section{ACKNOWLEDGMENTS}

S. K. Zh. gratefully acknowledges support from the European Research Council under the European Union's Seventh Framework Programme (FP7/2007-2013) / ERC Grant agreement 267499.

${ }^{1}$ H. Thomas, G. E. Morfill, V. Demmel, J. Goree, B. Feuerbacher, and D. Möhlmann, Phys. Rev. Lett. 73, 652 (1994).

${ }^{2}$ Y. Hayashi and K. Tachibana, Jpn. J. Appl. Phys. 33, L804 (1994).

${ }^{3}$ J. H. Chu and I Lin, Phys. Rev. Lett. 72, 4009 (1994).

${ }^{4}$ V. Nosenko, A. V. Ivlev, G. E. Morfill, Phys. Rev. Lett. 108, 135005 (2012). 
${ }^{5}$ S. Nunomura, J. Goree, S. Hu, X. Wang, A. Bhattacharjee, and K. Avinash, Phys. Rev. Lett. 89, 035001 (2002).

${ }^{6}$ A. Piel, V. Nosenko, J. Goree, Phys. Rev. Lett. 89, 085004 (2002).

${ }^{7}$ S. K. Zhdanov, S. Nunomura, D. Samsonov, and G. Morfill, Phys. Rev. E 68, 035401 (2003).

${ }^{8}$ K. Avinash, P. Zhu, V. Nosenko, and J. Goree, Phys. Rev. E 68, 046402 (2003).

${ }^{9}$ H. M. Thomas and G. E. Morfill, Nature (London) 379, 806 (1996).

${ }^{10}$ V. Nosenko, S. K. Zhdanov, A. V. Ivlev, C. A. Knapek, and G. E. Morfill, Phys. Rev. Lett. 103, 015001 (2009).

${ }^{11}$ A. Melzer, A. Schella, J. Schablinski, D. Block, and A. Piel, Phys. Rev. E 87, 033107 (2013).

${ }^{12}$ S. Nunomura, D. Samsonov, S. Zhdanov, and G. Morfill, Phys. Rev. Lett. 96, 015003 (2006).

${ }^{13}$ V. Nosenko and J. Goree, Phys. Rev. Lett. 93, 155004 (2004).

${ }^{14}$ A. Gavrikov, I. Shakhova, A. Ivanov, O. Petrov, N. Vorona, and V. Fortov, Phys. Lett. A 336, 378 (2005).

${ }^{15}$ P. Hartmann, M. C. Sándor, A. Kovács, and Z. Donkó, Phys. Rev. E 84, 016404 (2011).

${ }^{16}$ V. Nosenko, S. K. Zhdanov, A. V. Ivlev, G. E. Morfill, J. Goree, and A. Piel, Phys. Rev. Lett. 100, 025003 (2008).

${ }^{17}$ E. Thomas, J. D. Williams, and J. Silver, Phys. Plasmas 11, L37 (2004).

${ }^{18}$ S. Käding and A. Melzer, Phys. Plasmas 13, 090701 (2006).

${ }^{19}$ M. Kroll, S. Harms, D. Block, and A. Piel, Phys. Plasmas 15, 063703 (2008).

${ }^{20} \mathrm{M}$. Kroll, L. Mühlfeld, and D. Block, IEEE Transactions on Plasma Science 38, 897 (2010)

${ }^{21}$ D. D. Goldbeck, "Analyse Dynamischer Volumenprozesse in komplexen Plasmen", Ph.D. dissertation (Ludwig-MaximiliansUniversität München, 2003).

${ }^{22}$ D. Samsonov, A. Elsaesser, A. Edwards, H. M. Thomas, and G. E. Morfill, Rev. Sci. Instrum. 79, 035102 (2008).

${ }^{23}$ C. Killer, M. Himpel, and A. Melzer, Rev. Sci. Instrum. 85, 103711 (2014).

${ }^{24}$ L. Couëdel, V. Nosenko, S. K. Zhdanov, A. V. Ivlev, H. M. Thomas, and G. E. Morfill, Phys. Rev. Lett. 103, 215001 (2009).

${ }^{25} \mathrm{G}$. Lippmann, Comptes Rendus de l'Academie des Sciences 146, 446 (1908).

${ }^{26}$ P. Hartmann, I. Donkó, and Z. Donkó, Rev. Sci. Instrum. 84, 023501 (2013).

${ }^{27}$ Marketed by Raytrix GmbH, Schauenburgerstrasse 116, D-24118 Kiel, Germany.

${ }^{28}$ L. Couëdel, V. Nosenko, A. V. Ivlev, S. K. Zhdanov, H. M. Thomas, and G. E. Morfill, Phys. Rev. Lett. 104, 195001 (2010).

${ }^{29}$ T. G. Georgiev and A. Lumsdaine, "Depth of field in plenoptic cameras", In: P. Alliez, M. Magnor (eds.), Eurographics 2009 (2009), http://tgeorgiev.net/DepthOfField.pdf

${ }^{30}$ E. H. Adelson and J. Y. Wang, IEEE Transactions on Pattern Analysis and Machine Intelligence 14, 99-106 (1992).
${ }^{31}$ R. Ng, M. Levoy, M. Bredif, G. Duval, M. Horowitz, and P. Hanrahan, "Light field photography with a hand-held plenoptic camera", Technical Report No. 2005-02 (Stanford CTSR, 2005).

${ }^{32}$ A. Lumsdaine and T. Georgiev, "The Focused Plenoptic Camera", IEEE International Conference on Computational Photography, San Francisco, USA, 16-17 April 2009 (2009), DOI: 10.1109/ICCPHOT.2009.5559008

${ }^{33}$ C. Perwaß and L. Wietzke, Proc. SPIE 8291, 829108 (2012).

${ }^{34}$ M. Levoy and P. Hanrahan, "Light Field Rendering", Proc. of the 23rd Annual Conference on Computer Graphics, New Orleans, USA, August 4-9, 1996, 31-42 (1996), http://graphics.stanford.edu/papers/light/light-lorescorrected.pdf

${ }^{35}$ O. Johannsen, C. Heinze, B. Goldluecke, and C. Perwaß, "On the Calibration of Focused Plenoptic Cameras", in: Time-of-Flight and Depth Imaging: Sensors, Algorithms, and Applications, 302317 (Springer, Heidelberg, 2013), ISBN 978-3-642-44963-5.

${ }^{36}$ S. S. Rogers, T. A. Waigh, X. Zhao, and J. R. Lu, Physical Biology 4, 220-227 (2007).

${ }^{37}$ A. Heyden and M. Pollefeys, "Multiple View Geometry", in: Emerging Topics in Computer Vision, 45-107 (Prentice Hall, 2004), ISBN 978-0131013667.

${ }^{38}$ V. A. Schweigert, I. V. Schweigert, V. Nosenko, and J. Goree, Phys. Plasmas 9, 4465 (2002)

${ }^{39}$ M. Chaudhuri, V. Nosenko, C. Knapek, U. Konopka, A. V. Ivlev, H. M. Thomas, and G. E. Morfill, Appl. Phys. Lett. 100, 264101 (2012).

${ }^{40}$ C.-R. Du, V. Nosenko, S. Zhdanov, H. M. Thomas, and G. E. Morfill, EPL 99, 55001 (2012).

${ }^{41}$ A.Petersen, Raytrix GmbH, Private Communication (2015).

${ }^{42}$ Y. Feng, J. Goree, and B. Liu, Rev. Sci. Instrum. 78, 053704 (2007).

${ }^{43}$ B.F. Alexander, K.C. Ng, Opt. Eng.30, 13201331 (1991).

${ }^{44}$ R.D. Keane, R.J. Adrian, J Appl Sci Res 49, 191215 (1992).

${ }^{45}$ T. Roesgen, Experiments in Fluids 35, 252256 (2003).

${ }^{46}$ C. Durniak, D. Samsonov, J. F. Ralph, S. Zhdanov, and G. Morfill, Phys. Rev. E 88, 053101 (2013)

${ }^{47}$ S. Nunomura, J. Goree, S. Hu, X. Wang, and A. Bhattacharjee, Phys. Rev. E 65, 066402 (2002).

${ }^{48}$ S. K. Zhdanov, D. Samsonov, and G. E. Morfill, Phys. Rev. E 66, 026411 (2002).

${ }^{49}$ F. M. Peeters and X. Wu, Phys. Rev. A 35, 3109 (1987).

${ }^{50}$ L. Couëdel, S. K. Zhdanov, A. V. Ivlev, V. Nosenko, H. M. Thomas, and G. E. Morfill, Phys. Plasmas 18, 083707 (2011).

${ }^{51}$ D. Samsonov, S. Zhdanov, and G. Morfill, Phys. Rev. E 71, 026410 (2005).

${ }^{52}$ S. V. Vladimirov, P. V. Shevchenko, and N. F. Cramer, Phys. Rev. E 56, R74 (1997).

${ }^{53}$ D. H. E. Dubin, Phys. Rev. A 42, 4972 (1990).

${ }^{54}$ S. K. Zhdanov, A. Ivlev, and G. E. Morfill, Phys. Plasmas 16, 083706 (2009).

${ }^{55}$ T. B. Röcker, A. V. Ivlev, S. K. Zhdanov, and G. E. Morfill, Phys. Rev. E 89, 013104 (2014). 